\title{
Adaptation à la sécheresse et notion d'idéotype chez le blé dur. II. Caractères physiologiques d'adaptation
}

\author{
T Ali Dib $1^{*}$, P Monneveux ${ }^{2 *}$, JL Araus ${ }^{3}$ \\ 1 Université de Tichrine, faculté d'agronomie, Lattaquié, Syrie; \\ 2 ENSA-INRA, station d'amélioration des plantes, chaire de phytotechnie, 2 place Viala, 34060 Montpellier Cedex 01, France \\ 3 Universitat de Barcelona, facultat de biologia, departament de biologia, unitat de fisiologia vegetal, 08028 Barcelone, Espagne
}

(Reçu le 12 juillet 1991; accepté le 4 avril 1992)

\begin{abstract}
Résumé - Des populations locales de blé dur d'Algérie et du Moyen-Orient, et des variétés améliorées ont été comparées du point de vue de leurs paramètres phénologiques et morphophysiologiques d'adaptation à la sécheresse, l'accent étant mis sur des paramètres physiologiques liés à la transpiration, à l'osmorégulation et à la photosynthèse. Les résultats obtenus permettent de préciser les caractères physiologiques idéotypiques et le comportement adaptatif de chaque type de variété.
\end{abstract}

Triticum durum = blé dur / sécheresse / transpiration / osmorégulation / photosynthèse / idéotype

Summary - Adaptation to drought and ideotypes in durum wheat. II. Physiological traits. Durum wheat landraces from Algeria and the Middle East and improved varieties have been compared for phenological and different morphophysiological traits of adaptation to drought, those related to transpiration, osmoregulation, and photosynthesis being emphasized. The data obtained allow determination of ideotypic physiological traits and adaptive behaviour of each varietal type.

Triticum durum = durum wheat $/$ drought $/$ transpiration / osmoregulation / photosynthesis $/$ ideotype

\section{INTRODUCTION}

La difficulté d'expliquer le comportement d'un génotype de blé en situation de déficit hydrique à partir de ses seuls caractères d'enracinement a été évoquée par plusieurs auteurs (Hurd, 1973; Benlaribi et al, 1990); elle ressort également de l'étude précédente menée sur divers génotypes d'Afrique du Nord et du Moyen-Orient; ceci nous a donc conduit à envisager une étude plus complète des mécanismes d'adaptation, incluant divers paramètres phénologiques et morphophysiologiques parmi ceux décrits par Austin, 1987, Clarke, 1987 et Monneveux, 1991.

Les paramètres phénologiques d'adaptation, ou paramètres de précocité, définissent le calage de cycle vis-à-vis des contraintes de l'environnement; en intervenant sur ces paramètres, il est parfois possible d'éviter la coïncidence entre les stades critiques du développement et les dates d'occurrence maximale de certains accidents climatiques.

En conditions méditerranéennes par exemple, la recherche d'une plus grande précocité a été, jusqu'ici, le moyen le plus utilisé pour éviter les effets négatifs du déficit hydrique et des hautes températures de fin de cycle sur le remplissage du grain. Mais les limitations d'une telle stratégie ont été évoquées par plusieurs auteurs : réduction de la productivité (Laing et Fischer, 1977), risques accrus de gels tardifs (Single et Fletcher, 1979), réduction du développement racinaire (Derera et al, 1969).

Les paramètres morphophysiologiques d'adaptation permettent de rendre compte des phénomènes de tolérance (liés à la réduction

\footnotetext{
* Adresse actuelle : Chaire de phytotechnie-station d'amélioration des plantes, ENSA-INRA, 2 place Viala, 34060 Montpellier Cedex 01 (France).

** Correspondance et tirés à part.
} 
des pertes en eau, à la régulation des quantités d'eau absorbées, et à l'ajustement osmotique réalisé par l'accumulation de composés organiques et minéraux), et des phénomènes de résistance (aptitude à maintenir l'intégrité des structures et des fonctions). Au cours de ces dernières années, l'accent a en particulier été mis par différents auteurs sur les effets du déficit hydrique sur la photosynthèse, parmi lesquels on peut distinguer des effets stomatiques (Caemmerer et Farquhar, 1981) et des effets non stomatiques (Havaux et al, 1988). L'analyse des rapports photosynthèse/transpiration, et de l'impact d'un type donné de contrainte hydrique sur la photosynthèse permettent en effet d'aborder le problème des relations entre la tolérance à la sécheresse et la productivité (ou rendement potentiel en grains). De ce point de vue, des travaux récents (Blum, 1985; Febrero et al, 1990) ont également permis de mettre en évidence le rôle considérable joué par l'épi (glumes et barbes) dans la photosynthèse, et donc dans la production d'assimilats concourant au remplissage du grain.

Nous avons donc tenté, au cours de ce travail, d'analyser le fonctionnement de la plante, du point de vue des principales fonctions physiologiques concernées par l'adaptation au déficit hydrique : osmorégulation, photosynthèse, et transpiration.

L'étude a été conduite sur les mêmes génotypes que dans l'étude précédente concernant les caractères morphologiques d'enracinement, à savoir : des variétés issues de populations locales du Moyen-Orient et d'Algérie, et des variétés améliorées, productives, et à large adaptation : l'objectif de ce choix a été de dégager la notion d'idéotype morphophysiologique et de discuter la possibilité de définir, sur des bases physiologiques, des objectifs de sélection pour l'adaptation aux conditions environnementales.

\section{MATÉRIEL ET MÉTHODES}

\section{Matériel végétal}

La plupart des mesures ont porté sur 5 génotypes de blé dur : Haurani 27, Oued Zenati 368, Hedba 3, Sham 1 et Belikh 2, ayant fait précédemment l'objet d'études concernant les paramètres morphologiques racinaires de tolérance au déficit hydrique.

\section{Dispositif expérimental}

Les 5 génotypes ont été cultivés au champ et en serre.

Deux expérimentations au champ ont été conduites sur le domaine de Lavalette-ENSA.M (École nationale supérieure agronomique de Montpellier, France) au cours des années 1988-1989 et 1989-1990; les variétés ont été semées en parcelles de 5 lignes de $2 \mathrm{~m}$ $(20 \mathrm{~cm}$ entre les lignes) à raison de 4 parcelles par variété : 2 de ces 4 parcelles ont été conduites en sec (245 mm de pluie au cours du cycle en 1988-1989, $250 \mathrm{~mm}$ en 1989-1990), les 2 autres ont reçu des irrigations de complément correspondant à un total de 80 $\mathrm{mm}$.

Un premier essai en pot a, par ailleurs, été mené sur les 5 génotypes dans les serres de l'ENSA-INRA Montpellier (photopériode $15 \mathrm{~h}$, éclairement $75 \mathrm{~W} \cdot \mathrm{m}^{-2}$, température $20^{\circ} \mathrm{C}$ le jour et $15^{\circ} \mathrm{C}$ la nuit, humidité relative $70 \%$ ) : chaque pot en plastique est rempli de $3 \mathrm{~kg}$ de substrat composé de $80 \%$ de sable, $10 \%$ de terre et $10 \%$ de matière organique, et contient 5 plantes.

Un second essai en pots a porté uniquement sur Haurani 27 et Oued Zenati 368, les 2 populations locales les plus cultivées au Moyen-Orient et en Algérie respectivement. Cet essai a été mené sur les terrains expérimentaux de la faculté de biologie de l'université de Barcelone (Espagne). Les variétés ont été semées sur compost, dans des pots en plastique (diamètre $170 \mathrm{~mm}$ ), à raison de 6 plantules par pot. Les plantes ont été arrosées chaque jour.

\section{Mesures}

Les nombreuses mesures effectuées ont pour objectif une meilleure compréhension des spécificités de comportement de chaque génotype et sont décrites sur le tableau I.

Les composantes du rendement, les paramètres phénologiques, ainsi qu'un certain nombre de paramètres morphologiques ont été mesurés au champ (domaine de l'ENSA.M) sur les 5 génotypes. L'essai, conduit en serre sur les mêmes génotypes, avait, quant à lui, pour objectif principal l'étude des paramètres d'osmorégulation. Les paramètres physiologiques liés à la transpiration et à la photosynthèse ont été étudiés sur les variétés Oued Zenati 368 et Haurani 27 à l'université de Barcelone.

Le dosage des cires épicuticulaires a été effectué sur la dernière feuille bien développée des variétés Haurani 27 et Oued Zenati 368, au stade 4 feuilles, puis à l'anthèse : les cires sont extraites par agitation pendant $15 \mathrm{~s}$ dans le chloroforme (Mayeux et al, 1981) et l'extrait est ensuite séché à $50^{\circ} \mathrm{C}$ jusqu'à stabilisation de la masse (Febrero et al, 1989).

La proline est dosée par la méthode de Troll et Lindsley, 1955, modifiée par Benlaribi et Monneveux, 
Tableau I. Liste des mesures effectuées dans le cadre de l'étude.

\begin{tabular}{|c|c|c|}
\hline Mesure & Unité & Méthode (référence) \\
\hline \multicolumn{3}{|l|}{ Composantes du rendement 1} \\
\hline Biomasse & q.ha-1 & \\
\hline Rendement en grains & q.ha-1 & \\
\hline Indice de récolte & -- & \\
\hline Nb épis/plante & -- & \\
\hline Nb grains/épi & -- & \\
\hline Pds 1000 grains & $g$ & \\
\hline \multicolumn{3}{|l|}{ Paramètres phénologiques ${ }^{\dagger}$} \\
\hline Durée semis-épiaison & j & \\
\hline Durée semis-floraison & $\dot{j}$ & \\
\hline Durée semis-maturité & & \\
\hline Durée remplissage grain & j & \\
\hline \multicolumn{3}{|l|}{ Paramètres morphologiques } \\
\hline \multicolumn{3}{|l|}{ Stade 4 feuilles } \\
\hline Surface dernière feuille ${ }^{3}$ & $\mathrm{~cm}^{2}$ & \\
\hline Largeur dernière feuille $^{3}$ & $\mathrm{~mm}$ & \\
\hline Masse spéc dernière feuille 3 & g.cm-2 & \\
\hline Fréquence stomatique 3 & $\mathrm{~mm}^{-2}$ & \\
\hline Dépôt de cires épicuticulaires ${ }^{3}$ & mg.m-2 & Mayeux et al (1981) \\
\hline \multicolumn{3}{|l|}{ Stade anthèse } \\
\hline Port dernière feuille 1 & -- & \\
\hline Couleur feuilles 1 & -- & \\
\hline Surface dernière feuille ${ }^{3}$ & $\mathrm{~cm}^{2}$ & \\
\hline Largeur dernière feuille ${ }^{3}$ & $\mathrm{~mm}$ & \\
\hline Fréquence stomatique & $\mathrm{mm}^{-2}$ & \\
\hline Dépôt de cires épicuticulaires & $\mathrm{mg} \cdot \mathrm{m}^{-2}$ & Mayeux et al (1981) \\
\hline \multicolumn{3}{|l|}{ Maturité } \\
\hline Hauteur plante 1 & $\mathrm{~cm}$ & \\
\hline Longueur dernier entre-nœud 1 & $\mathrm{~cm}$ & \\
\hline Longueur du col de l'épi 1 & $\mathrm{~cm}$ & \\
\hline Longueur de l'épi 1 & $\mathrm{~cm}$ & \\
\hline Longueur des barbes 1 & $\mathrm{~cm}$ & \\
\hline Surface développée des barbes 1 & $\mathrm{~cm}^{2}$ & \\
\hline \multicolumn{3}{|l|}{$\begin{array}{l}\text { Paramètres physiologiques } \\
\text { Stade } 4 \text { feuilles }\end{array}$} \\
\hline Accumulation proline 2 & $\mu g \cdot g^{-1} M F$ & Troll et Lindsley (1955) \\
\hline Accumulation sucres solubles 2 & $\mu g . g^{-1} \mathrm{MF}$ & Schields et Burnett (1960) \\
\hline Potentiel hydrique foliaire 2 & $\mathrm{MPa}$ & Renard et Ndayishimie (1982) \\
\hline Conductance épidermique 3 & $\mathrm{mmol} \cdot \mathrm{m}^{-2} \cdot \mathrm{s}^{-1}$ & Rawson et Clarke (1988) \\
\hline Assimilation nette $\mathrm{CO}_{2}{ }^{3}$ & $\mu \mathrm{mol} . \mathrm{m}^{-2} \cdot \mathrm{s}^{-1}$ & \\
\hline Conductance stomatique $\mathrm{CO}_{2}{ }^{3}$ & $\mu \mathrm{mol} \cdot \mathrm{m}^{-2} \cdot \mathrm{s}^{-1}$ & Caemmerer et Farquhar (1981) \\
\hline Concentration intercellulaire & $\mu \mathrm{mol} .1^{-1}$ & Caemmerer et Farquhar (1981) \\
\hline Conductance résiduelle $\mathrm{CO}_{2}{ }^{3}$ & $\mu \mathrm{mol} . \mathrm{I}^{-1}$ & \\
\hline Inhibition fluorescence chlorophyllienne & $\%$ & Havaux et al (1988) \\
\hline \multicolumn{3}{|l|}{ Stade anthèse } \\
\hline Conductance épidermique ${ }^{3}$ & $\mu \mathrm{mol} . \mathrm{m}^{-2} . \mathrm{s}^{-1}$ & Rawson et Clarke (1988) \\
\hline Assimilation nette $\mathrm{CO}_{2}{ }^{3}$ & $\mu \mathrm{mol} . \mathrm{m}^{-2} \cdot \mathrm{s}^{-1}$ & \\
\hline Conductance stomatique $\mathrm{CO}_{2}{ }^{3}$ & $\mu \mathrm{mol} . \mathrm{m}^{-2} . \mathrm{s}^{-1}$ & Caemmerer et Farquhar (1981) \\
\hline Conductance intercellulaire & $\mu \mathrm{mol} . \mathrm{I}^{-1}$ & Caemmerer et Farquhar (1981) \\
\hline Conductance résiduelle $\mathrm{CO}_{2}{ }^{3}$ & $\mu \mathrm{mol} . \mathrm{m}^{-2} \cdot \mathrm{I}^{-1}$ & \\
\hline
\end{tabular}

\footnotetext{
${ }^{1}$ Au champ, domaine ENSA de Lavalette (Montpellier, France); ${ }^{2}$ en pot, sous serre à Montpellier; ${ }^{3}$ en pot, à l'université de Barcelone (Espagne) (voir dispositif expérimental).
} 
1988 (Le dosage est effectué sur quatre répétitions par traitement).

Le potentiel hydrique est mesuré à la presse à membrane, selon la technique de Renard et Ndayishimie, 1982 (mesures sur quatre répétitions pour chaque traitement).

Les mesures de potentiel hydrique, de turgescence relative, et d'inhibition des courbes d'induction de la fluorescence chlorophyllienne, ainsi que les dosages de proline ont été effectués sur la dernière feuille, au stade 4 feuilles, après 6,12 et $18 \mathrm{j}$ de déficit hydrique, et sur les 5 génotypes.

La conductance épidermique a été calculée à partir de la transpiration épidermique en utilisant les équations standard de diffusion (Coombs et al, 1985). Pour la mesure de la transpiration épidermique, les feuilles et les épis ont été amenés à turgescence maximale : leur extrémité basale a été immergée dans de l'eau déminéralisée et l'ensemble de l'organe placé sous sac plastique, pendant une nuit à l'obscurité; ils ont ensuite été déposés dans des boîtes de Petri, à l'intérieur d'une chambre climatisée pendant $2 \mathrm{~h}$ (température moyenne $24,5^{\circ} \mathrm{C}$, humidité relative voisine de $50 \%$ ).

Feuilles et épis ont été pesés à des intervalles de temps de $15 \mathrm{~min}$, afin d'établir une courbe de perte d'eau en fonction du temps : la perte d'eau est d'abord rapide, puis elle se stabilise de manière linéaire après 20 min environ autour d'un taux de perte épidermique (Hygen et Midgaard, 1954). La transpiration épidermique est estimée à partir de la pente de la droite audelà des 20 premières minutes d'expérience (Rawson et Clarke, 1988).

L'assimilation nette du $\mathrm{CO}_{2}$ par les feuilles est mesurée grâce à un appareil portable de mesure d'échanges gazeux (LCA-2, Analytical Development Co, Ltd, England), auquel est raccordée une chambre de mesure de Parkinson, en conditions lumineuses. La densité moyenne du flux de photons incident est restée comprise entre 1500 et $1700 \mu \mathrm{mol} . \mathrm{m}^{-2} . \mathrm{s}^{-1}$; l'humidité relative de l'air était de $50-60 \%$ et la température voisine de $25^{\circ} \mathrm{C}$.

La conductance stomatique du $\mathrm{CO}_{2}$ et la concentration intercellulaire en $\mathrm{CO}_{2}$ ont été calculées selon la méthode de Caemmerer et Farquhar, 1981.

La conductance résiduelle du $\mathrm{CO}_{2}$ a été mesurée à partir de la technique développée par Araus et Tapia, 1987.

L'efficience d'utilisation de l'eau a été évaluée en calculant le rapport du taux d'assimilation nette du $\mathrm{CO}_{2}$ au taux de transpiration.

L'inhibition des changements induits à la fluorescence chlorophyllienne a été mesurée selon la méthode de Havaux et al, 1988, à l'aide d'un fluorimètre SF30 (Brancker Ltd, Canada). Les mesures ont été effectuées sur la dernière feuille de plantes entières (soumises ou non à une contrainte hydrique); l'altération de la courbe de fluorescence induite a été étudiée en calculant à partir des courbes les valeurs du rapport $(P-S) / S(P=$ niveau maximum de fluorescence relative; $S=$ niveau pseudo-stationnaire de fluores- cence). Le rapport de fluorescence $(P-S)$ / $S$ est un paramètre empirique permettant de comparer la réponse des plantes au stress hydrique : c'est une mesure de la décroissance de l'intensité de fluorescence chlorophyllienne (ou extinction de la fluorescence) après le maximum $P$; l'extinction de la fluorescence est attribuée à une série de processus photochimiques et non-photochimiques, en particulier à la réoxydation des accepteurs primaires du PSII $\left(Q_{A}\right)$ réduits pendant les premiers moments d'illumination. Les valeurs obtenues sur plantes soumises au stress hydrique ont été comparées à celles obtenues dans le cas de plantes non stressées. Le rapport des valeurs de $(P-S) / S$ des échantillons stressés aux échantillons témoins est alors retenu comme un indicateur de la résistance chloroplastique des variétés testées, conformément aux conclusions de Ernez et Lannoye, 1991.

Les résultats présentés correspondent à la moyenne de 4 répétitions.

\section{RÉSULTATS}

Les résultats concernant les paramètres phénologiques (paramètres de précocité), le rendement et ses composantes, les paramètres morphologiques, et les paramètres physiologiques (osmorégulation, transpiration, photosynthèse) sont présentés successivement.

\section{Paramètres phénologiques}

Le tableau II, qui donne la longueur en jours des principales phases de cycle de développement pour les 5 variétés étudiées, met en évidence le caractère tardif des variétés algériennes (Oued Zenati 368 , Hedba 3 ), et la précocité des variétés locales et améliorées du Moyen-Orient.

\section{Rendement et composantes}

L'ensemble des caractères liés au rendement est donné par le tableau III.

En conditions irriguées, les rendements en grains des variétés améliorées (Belikh 2, Sham 1) apparaissent supérieurs à ceux des variétés locales : ces rendements élevés sont obtenus grâce à un indice de récolte élevé, un tallage plus élevé que chez les variétés locales algériennes, et un grain plus gros que chez la variété locale Haurani 27. La biomasse végétative produite par ces variétés améliorées est inférieure à celle des variétés locales algériennes, à paille haute. 
Tableau II. Paramètres phénologiques des 5 génotypes étudiés : longueur des principales phases de développement (moyenne des années 1988-1989 et 1989-1990).

\section{$\begin{array}{lllll}\text { Génotype } \quad H a u r a n i 27 & \text { Oued Zenati } 368 \text { Hedba } 3 & \text { Belikh } 2 & \text { Sham } 1\end{array}$}

Paramètres phénologiques *

\begin{tabular}{lccccc}
\hline Semis-épiaison & 128 & 136 & 133 & 126 & 125 \\
Semis-floraison & 137 & 144 & 142 & 136 & 135 \\
Semis-maturité & 160 & 168 & 167 & 161 & 158 \\
Phase de remplissage du grain & 23 & 24 & 25 & 25 & 23 \\
\hline
\end{tabular}

* Durées en jours.

L'absence d'irrigation se traduit par une diminution du rendement en grains, plus faible chez les variétés Sham $1(-20,5 \%)$ et Oued Zenati $368(-9,1 \%)$ que chez les autres variétés $(-31 \%$ en moyenne) : chez ces premières variétés, ceci s'explique en particulier par une faible variation du poids de 1000 grains $(-4,9 \%$ chez Oued Zenati, $-3,1 \%$ chez Sham 1); chez la variété Oued Zenati, la production de biomasse totale réagit très fortement à l'apport d'eau, à tel point que l'indice de récolte est plus élevé en l'absence d'irrigation $(0,26)$ qu'en conditions irriguées $(0,22)$.

\section{Paramètres morphologiques}

Les paramètres morphologiques des différentes variétés ont été comparés à 3 stades du développement : stade 4 feuilles, anthèse, et maturité; les 2 premières séries d'observations ont été réalisées sur Oued Zenati 368 et Haurani 27 à l'université de Barcelone, et les observations à maturité ont été effectuées au champ à Montpellier.

Les mesures effectuées au stade 4 feuilles (tableau IV) mettent en évidence certaines parti-

Tableau III. Composantes du rendement chez les 5 génotypes étudiés : le premier chiffre correspond aux parcelles irriguées et le second aux parcelles non irriguées (moyenne des années 1988 - 1989 et 1989 - 1990).

Génotype Haurani 27 Oued Zenati $368 \quad$ Hedba $3 \quad$ Belikh $2 \quad$ Sham 1

Composante du rendement

$\begin{array}{lccccc}\text { Biomasse aér tot (q.ha-1) } & 65,4 / 77,5 & 157,6 / 117,3 & 138,5 / 111,3 & 131,3 / 103,5 & 126,2 / 105,0 \\ \text { Rdt grains (q.ha-1) } & 29,8 / 22,1 & 34,1 / 31,0 & 45,0 / 27,9 & 47,0 / 33,3 & 52,3 / 41,3 \\ \text { Indice tallage 1 } & 3,4 / 3,0 & 2,6 / 2,2 & 3,0 / 2,6 & 3,7 / 3,0 & 3,9 / 3,3 \\ \text { Nb grains/épi } & 43,5 / 39,0 & 50,0 / 37,0 & 43,0 / 33,0 & 45,0 / 37,0 & 46,0 / 40,0 \\ \text { Poids 1 000 grains } & 38,7 / 38,0 & 51,5 / 49,0 & 50,0 / 48,0 & 45,8 / 41,5 & 43,3 / 42,0 \\ \text { Indice de récolte }{ }^{2} & 0,31 / 0,29 & 0,22 / 0,26 & 0,32 / 0,25 & 0,36 / 0,32 & 0,41 / 0,39\end{array}$

1 = nombre de talles par plante; ${ }^{2}=$ rapport de la masse du grain à la masse de substance sèche totale des parties aériennes à la récolte. 
Tableau IV. Paramètres morphologiques mesurés au stade 4 feuilles chez les génotypes Haurani 27 et Oued Zenati 368 : caractéristiques de la dernière feuille.

Génotype

Haurani 27 Oued Zenati 368

Paramètres

morphologiques

$\begin{array}{lrr}\text { Surface foliaire }\left(\mathrm{cm}^{2}\right) & 6,9 \pm 1,3 & 12,0 \pm 1,2 \\ \text { Largeur feuille }(\mathrm{mm}) & 6,8 \pm 0,4 & 8,4 \pm 0,4 \\ \text { Masse spéc }\left(\mathrm{g} \cdot \mathrm{m}^{-2}\right) & 33,1 \pm 0,4 & 37,6 \pm 4,7 \\ & & \\ \text { Fréquence stomatique }\left(\mathrm{mm}^{-2}\right) & \\ \text { Surface adaxiale } & 37,0 \pm 3,0 & 48,0 \pm 5,0 \\ \text { Surface abaxiale } & 34,0 \pm 5,0 & 40,0 \pm 6,0 \\ \text { Cires épicut }\left(\mathrm{mg} \cdot \mathrm{m}^{-2}\right) & 820,0 & 768,0\end{array}$

cularités de la variété Oued Zenati 368, telles que des feuilles plus longues et plus larges que chez Haurani 27; la fréquence de stomates est significativement plus élevée à la face adaxiale chez Oued Zenati 368; par contre les quantités de cires épicuticulaires par unité de surface de feuilles sont voisines chez les 2 variétés à ce stade.

La largeur et la surface de la feuille étendard sont, à l'anthèse, significativement plus importantes chez Oued Zenati 368 que chez Haurani 27 (tableau V); la masse spécifique de la feuille est par contre significativement plus élevée chez Haurani 27. Les fréquences stomatiques sont, à ce stade, très voisines chez les 2 variétés. Les quantités de cires épicuticulaires sont beaucoup plus importantes chez Haurani 27 que chez Oued Zenati 368.

À maturité (tableau VI), la hauteur de la plante est nettement plus élevée chez Oued Zenati 368 et Hedba 3 que chez les autres génotypes; chez Oued Zenati, cette taille élevée est associée à un dernier entre-nœud et un col de l'épi très long, ainsi qu'à des barbes très longues. Enfin, la longueur de l'épi est beaucoup plus réduite chez Haurani 27 que chez les autres génotypes.

\section{Paramètres physiologiques}

De très nombreux composés organiques et minéraux interviennent dans l'ajustement osmoti-
Tableau V. Paramètres morphologiques mesurés à l'anthèse (caractéristiques de la dernière feuille à l'anthèse).

Génotype

Haurani 27 Oued Zenati 368

Paramètres

morphologiques

$\begin{array}{lll}\text { Surfuce foliaire }\left(\mathrm{cm}^{2}\right) & 33,8 \pm 2,8 & 54,9 \pm 3,1 \\ \text { Largeur feuille }(\mathrm{mm}) & 18,8 \pm 0,6 & 21,2 \pm 0,7 \\ \text { Masse spéc }\left(\mathrm{g} \cdot \mathrm{m}^{-2}\right) & 56,0 \pm 0,9 & 50,2 \pm 0,3\end{array}$

Fréquence stomatique $\left(\mathrm{mm}^{-2}\right)$

$\begin{array}{lrr}\text { Surface adaxiale } & 62,0 & 51,0 \\ \text { Surface abaxiale } & 59,0 & 62,0 \\ & & \\ \text { Cires épicut }\left(\mathrm{mg} \cdot \mathrm{m}^{-2}\right) & 393,0 & 78,0\end{array}$

que, et nous nous limiterons, dans le cadre de cette étude, à la proline et aux sucres solubles :

- en ce qui concerne la proline (fig 1a), on constate que les teneurs augmentent fortement au début de la période de contrainte hydrique, pour diminuer après quelques jours de déficit (12 j chez Haurani 27, Belikh 2 et Hedba 3, 18 j chez Oued Zenati 368 et Sham 1); les teneurs les plus élevées peuvent être notées chez les variétés du Moyen-Orient : Haurani 27, Belikh 2, Sham 1;

- en ce qui concerne les sucres solubles (fig 1b), on constate que les variations de teneurs sont beaucoup plus faibles que dans le cas de la proline; les teneurs les plus élevées sont obtenues, au $12^{\ominus}$ j de déficit hydrique, par les variétés Oued Zenati 368 et Sham 1.

La conductance épidermique a été étudiée chez Oued Zenai 368 et Haurani 27 (tableau VII), grâce à des mesures de transpiration épidermique (celle-ci correspondant à la somme de la transpiration cuticulaire et de la transpiration stomatique liée à une fermeture incomplète des stomates). La conductance épidermique est plus faible chez Oued Zenati 368 que chez Haurani 27; toutefois, si l'on considère la transpiration épidermique totale de la dernière feuille, les valeurs sont alors beaucoup plus élevées pour la variété algérienne $\left(2,1.10^{-2}\right.$ et $10,9 \mathrm{mmol}^{-1}$ au stade 4 feuilles et à l'anthèse respectivement) que pour la variété du Moyen-Orient $\left(1,4.10^{-2}\right.$ et

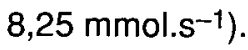


Tableau VI. Paramètres morphologiques à maturité (moyenne des années 1988 - 1989 et 1989 - 1990).

Génotype Haurani 27 Oued Zenati 368 Hedba $3 \quad$ Belikh $2 \quad$ Sham 1

Paramètre

morphologique

\begin{tabular}{lccccc}
\hline Hauteur plante $(\mathrm{cm})$ & 96,0 & 125,0 & 110,0 & 90,0 & 83,0 \\
Longueur dernier entre-nœud $(\mathrm{cm})$ & 17,0 & 19,5 & 17,0 & 18,0 & 18,0 \\
Longueur col épi (cm) & 44,0 & 49,0 & 40,0 & 44,0 & 33,0 \\
Longueur épi (cm) & 7,0 & 8,7 & 9,1 & 9,0 & 8,0 \\
Longueur barbes $(\mathrm{cm})$ & 8,0 & 16,0 & 11,0 & 13,0 & 11,5 \\
Surface développée barbes $\left(\mathrm{cm}^{2}\right)$ & 27,22 & 74,5 & - & - & - \\
Couleur barbes & Blanche & Noire foncée & Blanche & Blanche & $\begin{array}{c}\text { Noire } \\
\text { assez foncée }\end{array}$ \\
\hline
\end{tabular}

Parmi les paramètres liés aux échanges gazeux photosynthétiques, mesurés au stade 4 feuilles, la conductance stomatique du $\mathrm{CO}_{2}$, la conductance résiduelle du $\mathrm{CO}_{2}$, et le taux d'assi- milation nette du $\mathrm{CO}_{2}$ présentent des valeurs plus élevées chez Haurani 27 que chez Oued Zenati 368. Bien que la variété Haurani 27 présente une conductance stomatique plus élevée,

Tableau VII. Paramètres physiologiques mesurés au stade 4 feuilles et à l'anthèse sur les deux génotypes: Haurani 27 et Oued Zenati 368.

Paramètre

\section{Génotypes}

Conductance épidermique ( $\left.\mathrm{mmol} . \mathrm{m}^{-2} . \mathrm{s}^{-1}\right)$

$4^{e}$ feuille (st 4 f)

dernière feuille à l'anthèse

épi, à l'anthèse (mmol.g.ms.s ${ }^{-1}$ )

Assimilation nette du $\mathrm{CO}_{2}\left(\mu \mathrm{mol} . \mathrm{m}^{2} . \mathrm{s}^{-1}\right)$

$$
4^{e} \text { feuille (st } 4 \mathrm{f} \text { ) }
$$

dernière feuille à l'anthèse

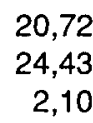

$16,2 \pm 2,9$

$14,9 \pm 0,5$

$13,0 \pm 0,8$

$9,6 \pm 0,7$

Conductance stomatique du $\mathrm{CO}_{2}\left(\mu \mathrm{mol} . \mathrm{m}^{-2} . \mathrm{s}^{-1}\right)$

$4^{e}$ feuille (st $4 \mathrm{f}$ )

dernière feuille à l'anthèse

$303,0 \pm 65,0$

$340,0 \pm 22,0$

$258,0 \pm 37,0$

$182,0 \pm 12,0$

Concentration intercellulaire du $\mathrm{CO}_{2}\left(\mu / . .^{-1}\right)$

4 feuille (st $4 \mathrm{f}$ )

dernière feuille à l'anthèse

$193,0 \pm 9,0$

$113,0 \pm 6,0$

$182,0 \pm 12,0$

$68,0 \pm 9,0$

Efficience de la transpiration 1

$4^{e}$ feuille (st $4 \mathrm{f}$ )

$3,01 \pm 0,25$

$4,04 \pm 0,10$

\footnotetext{
${ }^{1}$ Rapport du taux d'assimilation du $\mathrm{CO}_{2}$ au taux de transpiration.
} 


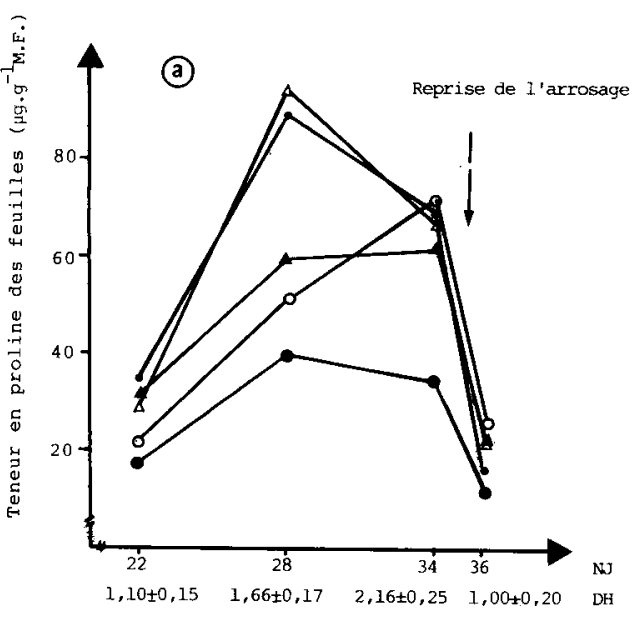

NJ: Nambre de jours après la levée. DH: Intensité correspondante du déficit hyarique (exprine par la moyenne des potentiels hydriques des 5 génotypes, en M.Pa).

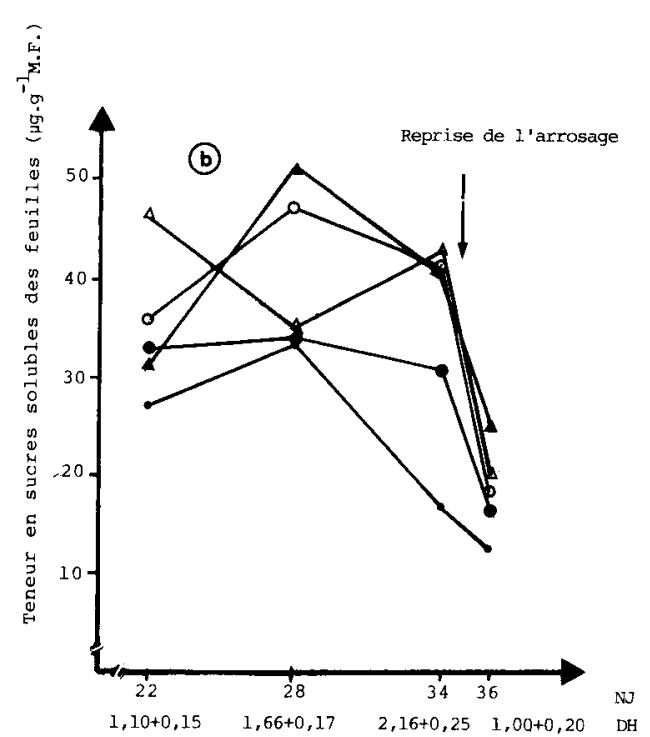

Fig 1. Accumulation de la proline et des sucres solubles au cours du déficit hydrique. a) proline. b) sucres solubles. L'analyse de variance met en évidence, au $28^{\theta}$ j après la levée (après $12 \mathrm{j}$ du stress hydrique):

- pour l'accumulation de proline: un effet variété $(P=0,0001)$, un effet traitement $(P=0,0001)$ et une interaction variété $\times$ traitement $(P=0,0001)$ hautement significatifs;

- pour l'accumulation de sucres solubles : un effet variété $(P=0,0144)$ significatif, un effet traitement $(P=0,0001)$ hautement significatif, et une interaction variété $\mathrm{x}$ traitement $(P=0,0235)$ significative. ( $\triangle$ Haurani 27 ; O Oued Zenati $368 ; \bullet$ Hedba $3 ; \cdot$ Belikh $2 ; \boldsymbol{A}$ Sham 1).

les concentrations intercellulaires en $\mathrm{CO}_{2}$ sont similaires chez les 2 génotypes. L'assimilation nette du $\mathrm{CO}_{2}$ au niveau de l'ensemble de la dernière feuille est plus élevée chez Oued Zenati $\left(1,5610^{-2}\right.$ et $\left.5,2710^{-2} \mu \mathrm{mol}^{-s^{-1}}\right)$.

Parmi les paramètres liés aux échanges gazeux photosynthétiques, mesurés au stade 4 feuilles, la conductance stomatique du $\mathrm{CO}_{2}$, la conductance résiduelle du $\mathrm{CO}_{2}$, et le taux d'assimilation nette du $\mathrm{CO}_{2}$ présentent des valeurs plus élevées chez Haurani 27 que chez Oued Zenati 368. Bien que la variété Haurani 27 présente une conductance stomatique plus élevée, les concentrations intercellulaires en $\mathrm{CO}_{2}$ sont similaires chez les 2 génotypes. L'assimilation nette du $\mathrm{CO}_{2}$ au niveau de l'ensemble de la dernière feuille est plus élevée chez Oued Zenati $\left(1,5610^{-2}\right.$ et $5,2710^{-2} \mu \mathrm{mol}^{-1}$ au stade 4 feuilles et à l'anthèse respectivement), que chez Haurani $\left(1,1210^{-2}\right.$ et $\left.5,0410^{-2} \mu \mathrm{mol}^{-\mathrm{s}^{-1}}\right)$.

Les effets non stomatiques du déficit hydrique sur la photosynthèse, ont été évalués sur les 5 génotypes, en comparant les évolutions du rapport $(P-S)$ / $S$, au cours de la contrainte (fig 2) : les valeurs les plus élevées mesurées sur plante entière peuvent être enregistrées au début de la période de déficit hydrique chez les génotypes du Moyen-Orient Haurani 27, Belikh 2, et Sham 1. À la fin de la période de contrainte $\left(18^{\mathrm{e}} \mathrm{j}\right)$, on peut noter une très nette différence entre les valeurs obtenues par les génotypes Haurani 27 et Sham 1, et celles obtenues par Belikh 2 et les variétés algériennes.

\section{DISCUSSION}

Les paramètres phénologiques et morphologiques pris en compte au cours de cette étude permettent de confirmer les observations de Grignac, 1965, concernant les caractéristiques des écotypes locaux (ou sous-espèces) méditerraneum typicum (Afrique du Nord) et syriacum (Moyen-Orient) :

- la sous-espèce $T$ durum ssp syriacum représentée dans notre étude par Haurani 27 est en effet caractérisée par un feuillage étroit (tableaux IV et $V$ ) et peu abondant, des tiges courtes et un épi court (tableau VI), à petits grains (tableau III);

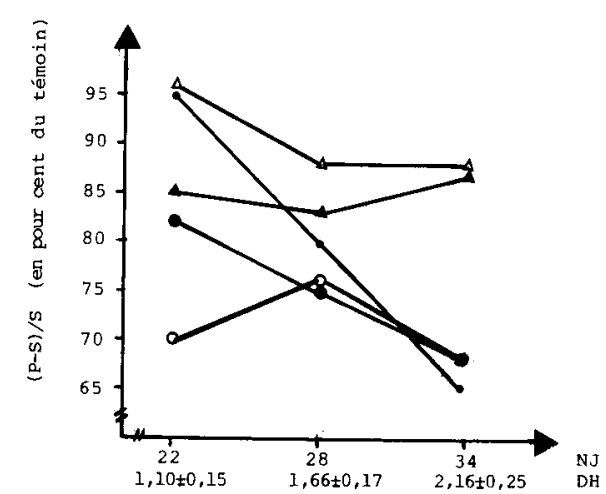

Fig 2. Évolution du rapport (P-S)/S (en \% du témoin) au cours du déficit hydrique chez les 5 génotypes du blé dur (mesure sur plante entière). L'analyse de variance révèle l'existence, au $34^{\mathrm{e}} \mathrm{j}$ après la levée (après $18 \mathrm{j}$ du stress hydrique), d'un effet variété $(P=0,020)$ et d'un effet traitement ( $P$ $=0,0013)$ significatifs, et d'une interaction variété $x$ traitement $(P=0,380)$ non significative ( $\Delta$ Haurani 27 ; O Oued $\mathrm{Ze-}$ nati 368; $\bullet$ Hedba 3; • Belikh 2; $\Delta$ Sham 1). 
la floraison et la maturation sont précoces (tableau II);

- l'écotype mediterraneum typicum représenté dans notre étude par les variétés locales Oued Zenatti 368 et Hebda 3, est caractérisé par un feuillage abondant (tableaux IV et V), des tiges longues et un épi long (tableau VI), à gros grains (tableau III).

L'étude des caractères morphologiques racinaires et de certains paramètres d'état de l'eau dans la plante, réalisée dans le cadre d'une étude précédente, et celle des paramètres physiologiques (osmorégulation, transpiration, photosynthèse) permettent de développer cette analyse et de compléter la description des comportements adaptatifs vis-à-vis de la sécheresse qui caractérisent les génotypes Haurani 27 et Oued Zenati 368 (fig 3).

La variété Haurani 27, caractérisée par une stratégie d'évitement grâce à son cycle de développement court, maintient également un potentiel hydrique élevé au cours du déficit hydrique. Or, le maintien d'un potentiel de l'eau élevé dans les feuilles est lié, d'après Turner, 1986 à une aptitude à extraire l'eau du sol et à une capacité à limiter les pertes d'eau par transpiration : on peut observer effectivement, chez la variété Haurani 27 , l'existence d'un système racinaire très déve-

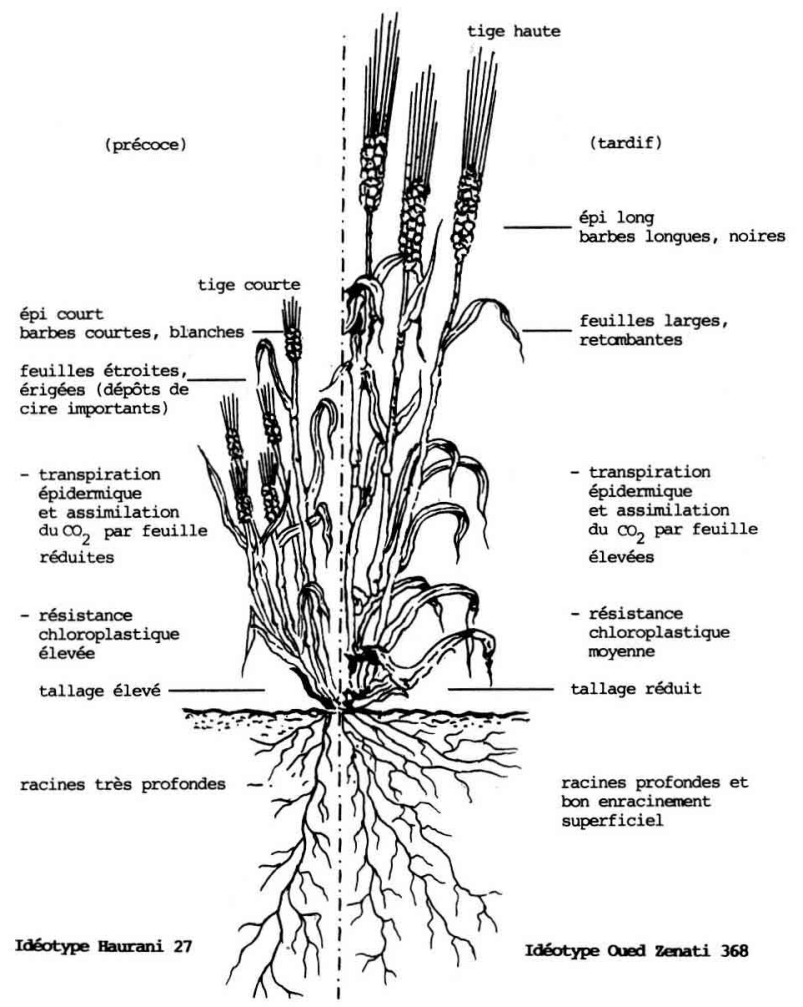

Fig 3. Représentation schématique des idéotypes de blé dur correspondant aux conditions du Moyen-Orient (Haurani 27) et des Hauts-Plateaux algériens (Oued Zenati 368). loppé, capable d'absorber l'eau des horizons les plus profonds, ainsi qu'un ensemble de caractéristiques morphophysiologiques concourant à réduire les pertes en eau : surfaces évapotranspirantes réduites (du fait du faible développement végétatif aérien et de la surface foliaire limitée), port érigé des feuilles, dépôt important de cires épicuticulaires sur la dernière feuille à l'anthèse (tableau V).

Les caractéristiques de l'épi (épi court, à barbes peu développées) contribuent également à une limitation des pertes en eau : la présence sur les glumes et les barbes non seulement de stomates, mais également de lenticelles (Febrero et al, 1989) lui confèrent en effet une conductance épidermique élevée, 4 fois plus importante environ que celle de la feuille étendard (Ali Dib et al, 1990). La réduction du rapport surface/ volume de l'épi peut dès lors apparaître comme un aspect essentiel dans la stratégie de réduction des pertes en eau, développée chez cette variété. Cette limitation des pertes par transpiration confère à cette variété une efficience de la transpiration élevée (tableau VII).

Les dosages d'osmolytes, limités à la proline et aux sucres solubles ne permettent pas d'expliquer de manière pertinente les phénomènes d'osmorégulation intervenant au cours du déficit hydrique chez les différentes variétés testées: on note toutefois que la variété Haurani 27 se caractérise par une accumulation de proline très importante en début de déficit hydrique; or, plusieurs auteurs ont évoqué le rôle joué par cet acide aminé dans la tolérance au déficit hydrique :

- Pearson et Stewart, 1986, indiquent que la proline pourrait jouer un rôle essentiel dans l'ajustement des pressions osmotiques cytoplasme-vacuole;

- Bellinger et Lahrer, 1987, admettent, quant à eux, que la proline pourrait avoir un rôle très important dans l'ajustement du métabolisme énergétique, sa synthèse étant très liée au métabolisme des sucres et à la respiration (Venkamp et al, 1989). Ces faits expliqueraient la relation étroite entre capacité d'accumulation de proline en cas de déficit hydrique et niveau de tolérance à la sécheresse, relevée chez les céréales, et en particulier chez le blé dur (Monneveux et Nemmar, 1986; Narayan et Misra, 1989).

Le rôle des sucres solubles dans l'ajustement osmotique a par ailleurs souvent été évoqué chez les céréales, en particulier par Vieira $\mathrm{Da}$ Silva, 1968, dans le cas du blé dur. 
La faible réduction de croissance des feuilles, observée en cas de déficit en eau sur la variété Haurani 27 dans le cadre du précédent article, et l'étroite corrélation négative notée par Blum, 1989 entre ce caractère et l'ajustement osmotique, tendent par ailleurs à confirmer sa capacité d'osmorégulation. Si elle se vérifiait au cours d'études futures, celle-ci permettrait d'expliquer les faibles variations de teneurs en eau enregistrées au cours du déficit hydrique, lorsqu'on la compare, par exemple, avec les variétés algériennes Oued Zenati 368 et Hedba 3.

La variété Haurani 27 se caractérise enfin par une faible décroissance de l'intensité de la fluorescence chlorophyllienne (fig 2). Ce résultat pourrait s'expliquer par une faible inhibition, dans les plantes stressées, de l'activité photochimique des chloroplastes qui peut être le résultat direct de la sécheresse sur le système photosynthétique de transport d'électrons ou la conséquence indirecte de l'inhibition du cycle de Calvin de fixation du $\mathrm{CO}_{2}$, par exemple en réponse à la fermeture des stomates (Ben et al, 1987; Cornic et Briantais, 1991).

En résumé, la variété Haurani 27 semble caractérisée par une stratégie de type conservatif dont les principaux éléments sont (fig 3) :

- un évitement (relatif) du déficit hydrique terminal, par raccourcissement du cycle;

- une tolérance avec maintien du potentiel hydrique foliaire, liée à une longueur maximale des racines élevée et à des caractéristiques morphophysiologiques conduisant à une limitation des pertes en eau (feuilles étroites, courtes, et érigées; biomasse aérienne réduite; épi et barbes courts; abondance de cires épicuticulaires);

- une capacité d'ajustement osmotique probablement très élevée, dont l'une des manifestations peut être recherchée dans l'accumulation de proline. Le maintien du potentiel hydrique et la capacité élevée d'osmorégulation contribuent à maintenir une teneur en eau élevée dans la plante (qui permet en particulier la poursuite de la croissance végétative);

- une résistance élevée, se traduisant par une capacité à préserver les structures et le fonctionnement de l'appareil photosynthétique à des teneurs en eau faibles.

Les variétés Oued Zenati et Hedba 3 présentent, quant à elles, une toute autre stratégie, qui semble correspondre aux contraintes environnementales propres à la zone dont elles sont originaires (Hauts-Plateaux algériens). Cette région présente en effet des risques élevés de gels tar- difs, et une sécheresse de type intermittente, surtout marquée en fin de cycle (Baldy, 1986) : l'occurrence des gels printaniers interdit la culture de variétés précoces (Mouret et al, 1991). Dans de telies conditions, l'idéotype doit être en mesure de répondre à plusieurs objectifs :

- traverser des périodes de déficit hydrique de durées limitées mais très variables; ceci peut être obtenu grâce à des mécanismes de régulation interne et de redistribution de l'eau vers les organes en croissance, comme le surdimensionnement du développement foliaire (Ben Salem et Vieira Da Silva, 1991) permettant des redistributions ultérieures de l'eau disponible vers les feuilles photosynthétiquement les plus actives, et la contribution possible du système racinaire très volumineux aux pertes en eau des parties aériennes (Benlaribi et al, 1990);

- supporter les déficits hydriques, et les hautes températures en fin de cycle : les barbes, très développées chez Oued Zenati 368 (tableau VI), pourraient jouer ici un rôle primordial. Bien que les barbes apparaissent peu efficaces en tant qu'organes de dissipation de la chaleur (Hatfield et al, 1984), leur photosynthèse est, selon Blum, 1985 , moins sensible à l'action inhibitrice des hautes températures que celle des glumes ou de la dernière feuille. Le rapport photosynthèse/ transpiration élevé et la thermostabilité de la photosynthèse de l'épi pourraient d'ailleurs être liées à l'existence, dans ces organes, d'un métabolisme de type intermédiaire $\mathrm{C}_{4}-\mathrm{C}_{3}$, évoqué par Ziegler-Jöns, 1989. De nombreux travaux, réalisés sur une large gamme de génotypes, semblent confirmer le rôle des barbes dans le remplissage du grain en conditions de déficit hydrique (Grignac, 1965; Hadjichristodoulou, 1985; Ali Dib et al, 1990);

- être en mesure de tirer partie des pluies de printemps grâce à un bon enracinement superficiel (Ali Dib et Monneveux, 1992).

Les variétés améliorées Sham 1 et Belikh 2 donnent les rendements les plus élevés en conditions hydriques favorables (tableau III) : cette productivité élevée s'explique essentiellement par un fort indice de récolte dû à un raccourcissement de la paille et à une fertilité de l'épi élevés; ces variétés donnent également des rendements élevés en conditions sèches, qu'il s'agisse des conditions de la France (tableau III) ou de celles de la Syrie (Nachit, 1987).

Les principaux paramètres morphophysiologiques qui pourraient expliquer ce comportement en conditions sèches sont : 
- une grande précocité (tableau II);

- le maintien d'un potentiel hydrique foliaire et d'une teneur en eau élevés au cours de la contrainte (Ali Dib et Monneveux, 1992);

- une teneur en sucres solubles élevée chez Sham 1 (fig $1 \mathrm{~b}$ ) et une teneur en proline élevée chez les deux variétés (fig 1a);

- une valeur élevée du quotient $(\mathrm{P}-\mathrm{S}) / \mathrm{S}$, chez la variété Sham 1 (fig 2); on note cependant une réduction de la valeur de $(P-S) / S$ au cours du temps chez Belikh 2, qui pourraient être rapprochée de l'abaissement plus rapide de la teneur en eau de cette variété.

Ces différents paramètres, ainsi que d'autres paramètres morphologiques déjà évoqués (longueur de l'épi, longueur des barbes, port et surface des feuilles) montrent que les variétés doivent être rapprochées de l'idéotype Haurani 27, adapté aux conditions du Moyen-Orient.

Bien que les informations disponibles actuellement soient encore insuffisantes pour juger du comportement des variétés Sham 1 et Belikh 2 en Algérie, les rendements obtenus au cours de cette étude suggèrent que ces variétés ne correspondent pas à l'idéotype souhaitable pour les Hauts-Plateaux : les enquêtes agronomiques réalisés par Mouret et al, 1991, dans la région de Sidi-Bel-Abbès révèlent d'ores et déjà la sensibilité extrême de la variété Sham 1 aux gels tardifs, du fait de sa précocité.

\section{CONCLUSION}

L'analyse systématique des caractères phénologiques et morphophysiologiques d'adaptation au déficit hydrique montre que le problème de la tolérance ne peut être envisagé indépendamment du type de sécheresse rencontré. Cette constatation implique que soit associé, à chaque combinaison particulière des contraintes environnementales, un idéotype spécifique, défini par un certain nombre de paramètres. Cette définition de l'idéotype à rechercher nous semble pouvoir s'inspirer de celle des écotypes locaux, résultat d'une sélection pluriséculaire sous la pression des contraintes environnementales, mais elle doit aussi chercher à dégager des objectifs d'amélioration de la productivité non antagonistes de la tolérance au déficit hydrique. La comparaison effectuée dans le cadre de cette étude entre écotypes du Moyen-Orient et écotypes des HautsPlateaux algériens n'est qu'une illustration de la démarche analytique explicative (Monneveux,
1991) permettant de mieux comprendre le fonctionnement du végétal dans un milieu donné; une définition plus précise des paramètres d'adaptation (et donc des objectifs ultérieurs de la sélection) requiert toutefois que cette approche soit associée à une approche synthétique multicaractères (Acevedo et Ceccarelli, 1989) permettant seule d'identifier et de hiérarchiser expérimentalement les caractères de tolérance associés à un type de sécheresse donné. La mise en place de programmes combinant les 2 approches devrait donc permettre d'orienter efficacement la sélection des céréales dans les zones à fortes contraintes.

\section{REFERENCES}

Acevedo E, Ceccarelli S (1989) Role of physiologist breeder in a breeding program for drought resistance conditions. In: Drought resistance in cereals (FWG Baker, ed) Oxon (UK), 117-139

Ali Dib T, Monneveux P, Araus JL (1990) Breeding durum wheat for drought tolerance analytical, synthetical approaches, and their connections. In: Symposium: wheat breeding-prospects and future approaches. Varna, Bulgaria, June 4-8, 1990 (I Panayotov, S Pavlova, eds) 224-240

Ali Dib, Monneveux P (1992) Adaptation à la sécheresse et notion d'idéotype chez le blé dur. I. Caractères morphologiques d'enracinement. Agronomie 12 ,

Araus JL, Tapia L (1987) Photosynthetic gas exchange characteristics of wheat flag leaf blades and sheath during grain filling. The case of a spring crop grown under Mediterranean climate conditions. Plant Physiol 85, 667-673

Austin RB (1987) Some crop characteristics of wheat and their influence on yield and water use. In: Drought tolerance in winter cereals (JP Srivastava, E Porceddu, E Acevedo, S Varma, eds) John Wiley and Sons, UK, 321-326

Baldy C (1986) Comportement des blés dans les climats méditerranéens. Ecol Mediterr, XII (3-4-, 7378

Bellinger $Y$, Larher F (1987) Proline accumulation in higher plants: a redox buffer? Life sci adv Plant Physiol 6, 23-27

Ben GY, Osmond CB, Sharkey TD (1987) Comparaisons of photosynthetic responses of Xanthium strumarim and Helianthus annuus to chronic and acute water stress in sun and shade. Plant Physiol 84, 476-482

Benlaribi M, Monneveux P (1988) Étude comparée du comportement, en situation de déficit hydrique, de deux variétés algériennes de blé dur (Triticum durum Desf) adaptées à la sécheresse. $C R$ Séances Acad Agric Fr 74 (5), 73-83 
Benlaribi M, Monneveux P, Grignac P (1990) Étude des caractères d'enracinement et de leur rôle dans l'adaptation au déficit hydrique chez le blé dur (Triticum durum Desf). Agronomie 10, 305-322

Ben Salem M, Vieira Da Silva JP (1991) Polymorphisme variétal de résistance à la sécheresse chez les céréales à paille : cas du blé. In : l'Amélioration des plantes pour l'adaptation aux milieux arides (AUPELF-UREF, ed) John Libbey Eurotext, Paris, 25-34

Blum A (1985) Photosynthesis and transpiration in leaves and ears of wheat and barley varieties. $J$ Exp Bot 36, 432-440

Blum A (1989) Osmotic adjustment and growth of barley genotypes under drought stress. Crop Sci 29 , 230-233

Caemmerer S Von, Farquhar GD (1981) Some relationships between the biochemistry of photosynthesis and the gas exchange of leaves. Planta 153 376-387

Clarke JM (1987) Use of physiological and morphological traits in breeding programmes to improve drought resistance of cereals. In: Drought tolerance in winter cereals (JP Srivastava, E Porceddu, E Acevedo, S Varma, eds) John Wiley and Sons, UK, 171-189

Coombs J, Hall DO, Long SP, Scurlock JMO (1985) Techniques in bioproductivity and photosynthesis. Pergamon Press, Oxford, 2nd edn

Cornic G, Briantais JM (1991) Partitionining of photosynthesis electron flow between $\mathrm{CO}_{2}$ and $\mathrm{O}_{2}$ reduction in a $\mathrm{C}_{3}$ leaf (Phasesolus vulgaris $\mathrm{L}$ ) at different $\mathrm{CO}_{2}$ concentrations and during drought stress. Planta 183, 178-184

Derera NF, Marshall DR, Balaam LN (1969) Genetic variability in root development in relation to drought tolerance in spring wheats. Exp Agric 5, 327-337

Ernez M, Lannoye R (1991) Quantification of physiological disorders in stressed plants. In: Physiologybreeding of winter cereals for stressed Mediterranean environments. Montpellier, France, 3-6 July 1989 (E Acevedo, AP Conesa, P Monneveux, J Srivastava, eds) Colloques INRA $n^{\circ} 55,414-433$

Febrero A, Vendrell P, Alegre L, Araus JL (1991) Epidermal conductance in flag leaves and ears of several durum wheat landraces and varieties: morphological and anatomical characteristics involved. In: Physiology breeding of winter cereals for stressed Mediterranean environments. Montpellier, France, 3-6 July 1989 (E Acevedo, AP Conesa, P Monneveux, J Srivastava, eds) Colloques INRA $n^{\circ}$ 55, 143-157

Febrero A, Bort J, Brown RH, Araus JL (1990) The role of durum wheat ear as photosynthetic organ during grain filling. In: Advanced trends in photosynthesis. Mallorca

Grignac P (1965) Contribution à l'étude de Triticum durum DESF. Thèse doctorat, Toulouse, $151 \mathrm{p}$

Hadjichristodoulou A (1985) Stability performance of cereals in low-rainfall areas as related to adaptative traits. In: Drought tolerance in winter cereals (JP Srivastava, E Porceddu, E Acevedo, S Varma, eds) John Wiley and Sons, UK, 191-200

Hatfield JL, Pinter PJ, Chasseray E, Ezra E, Reginato RJ, idso SB, Jackson RD (1984) Effects of panicles on infrared thermometer measurements of canopy temperature in wheat. Agric For Meteorol 32, 97 105

Havaux M, Ernez M, Lannoye R (1988) Sélection de variétés de blé dur (Triticum durum Desf) et de blé tendre (Triticum aestivum $\mathrm{L}$ ) adaptées à la sécheresse, par la mesure de l'extinction de la fluorescence de la chlorophylle in vivo. Agronomie 8, 193199

Hurd EA (1974) Phenotype and drought tolerance in wheat. Agric Météorol 14, 39-55

Hygen G, Midgaard E (1954) A reinvestigation of the influence of varying air humidity on cultivar transpiration in Pinus sylvestris L. Physiol Plant 7, 128-140

Laing DR, Fischer RA (1977) Adaptation of semi-dwarf wheat cultivars to rainfed conditions. Euphytica 26, 129-139

Mayeux JHS, Jordan WR, Meyer RE, Meola SM (1981) Epicuticular wax on golden weed (Isocoma spp) leaves: variation with species and season. Weed Sci 29, 389-393

Monneveux P (1991) Quelles stratégies pour l'amélioration génétique de la tolérance au déficit hydrique des céréales d'hiver? In: L'amélioration des plantes pour l'adaptation aux milieux arides (AUPELF UREF, ed) John Libbey Eurotext, Paris, 165-186

Monneveux P, Nemmar M (1986) Contribution à l'étude de la résistance à la sécheresse chez le blé tendre (Triticum aestivum $\mathrm{L}$ ) et chez le blé dur (Triticum durum DESF). Étude de l'accumulation de la proline au cours du cycle de développement. Agronomie 6, 583-590

Mouret JC, Conesa AP, Bouchier A, Gaid M, Oúld Said O (1991) Identification des facteurs de variabilité du rendement du blé dur en conditions hydriques limitantes dans la région de Sidi-Bel-Abbès, (Algérie). In: Physiology-breeding of winter cereals for stressed mediterranean environments. Montpellier, France, 3-6 July 1989 (E Acevedo, AP Conesa, $P$ Monneveux, J Srivastava, eds) Colloques INRA $n^{\circ} 55,35-48$

Nachit M (1987) Durum wheat breeding. In: Cereal improvement program. ICARDA Annu Rep 40-57

Narayan D, Misra RD (1989) Free proline accumulation and water-stress resistance in bread wheat (Triticum aestivum) and durum wheat ( $T$ durum). Indian J Agric Sci 59, 3, 176-178

Pearson J, Stewart GR (1986) Aspects of nitrogen metabolism in barley in relation to drought stress. Agriculture, drought resistance in plants physiological and genetic aspect. Comm Eur Commun 203-213

Rawson HM, Clarke JM (1988) Nocturnal transpiration in wheat. Aust J Plant Physiol 15, 397-406

Renard C, Ndayishimie V (1982) Étude des relations hydriques chez Coffea arabica L. I. Comparaison 
de la presse à membrane et de la chambre à pression pour la mesure du potentiel hydrique foliaire. Café Cacao Thé 26, 27-30

Schields R, Burnett W (1960) Determination of proteinbound carbohydrate in serum by a modification anthrone method. Anal Chem 32, 885-886

Single WV, Fletcher RJ (1979) Resistance of wheat to freezing in the heading stages. Proc 5th Int Wheat Genet Symp New Delhi, Feb 23-28, 1978 (S Ramanujam, ed) $\mathrm{t} 1,188-191$

Troll W, Lindsley J (1955) A photometric method for the determination of proline. J Biol Chem 215, 655660
Turner NC (1986) Adaptation to water deficits: a changing perspective. Aust J Plant Physiol 13, 175-190

Venekamp JH, Lampe JEM, Koot JTM (1989) Organic acids as sources for drought-induced proline synthesis in field bean plants (Vicia faba L). J Plant Physiol 133, 654-659

Vieira Da Silva J (1968) Influence of osmotic potential of the nutrient solution on the soluble carbohydrate and starch content of three species of Gossypium. C R Acad Sci Paris 267, 1289-1299

Ziegler-Jöns A (1989) Gaz exchange of ears of cereals in response to carbon'dioxide and light. I. Occurence of $\mathrm{a} \mathrm{C}_{3}-\mathrm{C}_{4}$ intermediate type of photosynthesis. Planta 178, 164-175 